BULL. AUSTRAL. MATH. SOC.

VOL. 25 (1982), 187-206.

\title{
ON RANDOMLY 3-AXIAL GRAPHS
}

\section{Yousef Alavi, Sabra S. Anderson, Gary Chartrand and S,F, Kapoor}

\begin{abstract}
A graph $G$, every vertex of which has degree at least three, is randomly 3-axial if for each vertex $v$ of $G$, any ordered collection of three paths in $G$ of length one with initial vertex $v$ can be cyclically randomly extended to produce three internally disjoint paths which contain all the vertices of $G$. Randomly 3-axial graphs of order $p \geq 4$ are characterized for $p$ 1 $1(\bmod 3)$, and are shown to be either complete graphs or certain regular complete bipartite graphs.
\end{abstract}

A graph $G$ was defined to be randomly traceable in Chartrand and Kronk [2], if, for each vertex $v$ of $G$, every path with initial vertex $v$ can be extended to a hamiltonian path with initial vertex $v$. Equivalently, a graph $G$ of order at least three is randomly traceable if every path of $G$ is contained in some hamiltonian cycle of $G$. It was proved in Chartrand and Kronk [2], that a graph $G$ of order $p$ is randomly traceable if and only if $G$ is isomorphic to $K_{p}, C_{p}$ or $K(p / 2, p / 2)$, where in the last case $p$ is even.

A random extension of a path $P: v_{1}, v_{2}, \ldots, v_{n}$ in a graph is a path $P^{*}: v_{1}, v_{2}, \ldots, v_{n}, v_{n+1}$ where $v_{n+1}$ is any vertex of the graph adjacent to $v_{n}$ that does not belong to $P$. A collection of paths, each

Received 9 September 1981. The second author wishes to express her appreciation to Western Michigan University for the hospitality extended to her as a Visiting Scholar during the period of this research.

187 
with initial vertex $v$, is called intemally disjoint if every two paths in the collection have only the vertex $v$ in common. Let $P=\left(P_{1}, P_{2}, P_{3}, \ldots, P_{k}\right)$ be an ordered collection of $k$ internaliy disjoint paths with $P_{i}: v, v_{1 i}, v_{2 i}, \ldots, v_{t i}, 1 \leq i \leq k$, such that each path has initial vertex $v$ and length $t \geq I$. By a cyclic random extension of $P$ we mean a sequence of random extensions of the paths $P_{1}, P_{2}, \ldots$ resulting in the following sequence of ordered collections of internally disjoint paths:

$$
\begin{array}{r}
\left(P_{1}^{*}, P_{2}, P_{3}, \ldots, P_{k}\right),\left(P_{1}^{*}, P_{2}^{*}, P_{3}, \ldots, P_{k}\right),\left(P_{1}^{*}, P_{2}^{*}, P_{3}^{*}, \ldots, P_{k}\right), \ldots, \\
\left(P_{1}^{*}, P_{2}^{*}, P_{3}^{*}, \ldots, P_{k}^{*}\right),\left(\left(P_{1}^{*}\right) *, P_{2}^{*}, P_{3}^{*}, \ldots, P_{k}^{*}\right), \\
\left(\left(P_{1}^{*}\right) *\left(P_{2}^{*}\right) *, P_{3}^{*}, \ldots, P_{k}^{*}\right) \ldots .
\end{array}
$$

In Burns, Chartrand, Kapoor and Saba [1], a graph $G$, where $1 \leq k \leq \delta(G)$, was called randomly $k$-axial if for each vertex $v$ of $G$, any ordered collection of $k$ paths in $G$ of length one having initial vertex $v$ can be cyclically randomly extended to produce $k$ internally disjoint paths which contain all the vertices of $G$.

It is proved in Burns, et al. [1], that a graph $G$ is

(i) randomly l-axial or

(ii) randomly 2-axial with $\delta(G) \geq 2$ if and only if $G$ is randomly traceable.

Thus, randomly $k$-axial graphs serve as a generalization of randomly traceable graphs. The problems of determining which bipartite graphs and which complete $n$-partite graphs are randomly $k$-axial were also studied there. Among the results presented were

(1) the complete bipartite graph $k\left(n_{1}, n_{2}\right)$ is randomly $k$-axial $(k \geq 3)$ if and only if $n_{1}=n_{2}$ and $n_{1} \equiv 0,1$ $(\bmod k)$ and

(2) the complete tripartite graph $K\left(n_{1}, n_{2}, n_{3}\right)$ is randomly $k$-axial $(k \geq 2)$ if and only if $n_{1}=n_{2}=n_{3}=k / 2$.

It is not difficult to verify that for $t \geq 3$, the regular complete 
$t$-partite graph $K(d, d, \ldots, d)$ is randomly $k$-axial for all $d \geq 1$ and $k=(t-1) d$. It was conjectured by Burns, et al. [1] that

(1) for $k \geq 2$ and $t \geq 3$, a noncomplete graph $K\left(n_{1}, n_{2}, \ldots, n_{t}\right)$ is randomly $k$-axial if and only if $n_{1}=n_{2}=\ldots=n_{t}=k /(t-1)$ and that

(2) every randomly $k$-axial graph $(k \geq 3)$ is a regular complete multipartite graph.

We have already noted that the randomly 1-axial graphs and randomly 2-axial graphs have been completely classified. From the results and conjectures stated above, it would follow that the only randomly 3-axial graphs are complete graphs of order at least 4 and certain complete bipartite graphs. We verify that such is the case in many instances. First we present an improvement of a result of Burns, et al. [1].

THEOREM 1. If $G$ is a randomly $k$-axial graph $(k \geq 3)$ of order $p$, where $p \equiv 0(\bmod k)$, then either

(i) $G \cong K_{p}$, or

(ii) $G \cong K(p / 2, p / 2)$ when $p / k$ is even.

Proof. Let $m=p / k$. If $m$ is even, then it was proved in Burns, et al. [1], that either $G \cong K_{p}$ or $G \cong K(p / 2, p / 2)$. Thus we assume $m$ is odd, and let $v_{0} \in V(G)$. Since $G$ is randomly $k$-axial, $\delta(G) \geq k$, and then $m \geq 3$. Now we can select any $k$ edges incident with $v_{0}$ to produce $k$ paths of length one having initial vertex $v_{0}$, and cyclically randomly extend these to obtain a spanning subgraph of $G$ (see Figure 1 ). The levels $L_{1}, L_{2}, \ldots, L_{m-1}$ and $L_{m}^{*}$, where

$$
L_{i}=\left\{v_{i 1}, v_{i 2}, \ldots, v_{i k}\right\}, 1 \leq i \leq m-1 \text {, }
$$

and

$$
L_{m}^{*}=\left\{v_{m 1}, v_{m 2}, \cdots, v_{m, k-1}\right\}
$$

are also indicated in Figure 1. Further define $L_{m}=L_{m}^{*} \cup\left\{v_{0}\right\}$. 


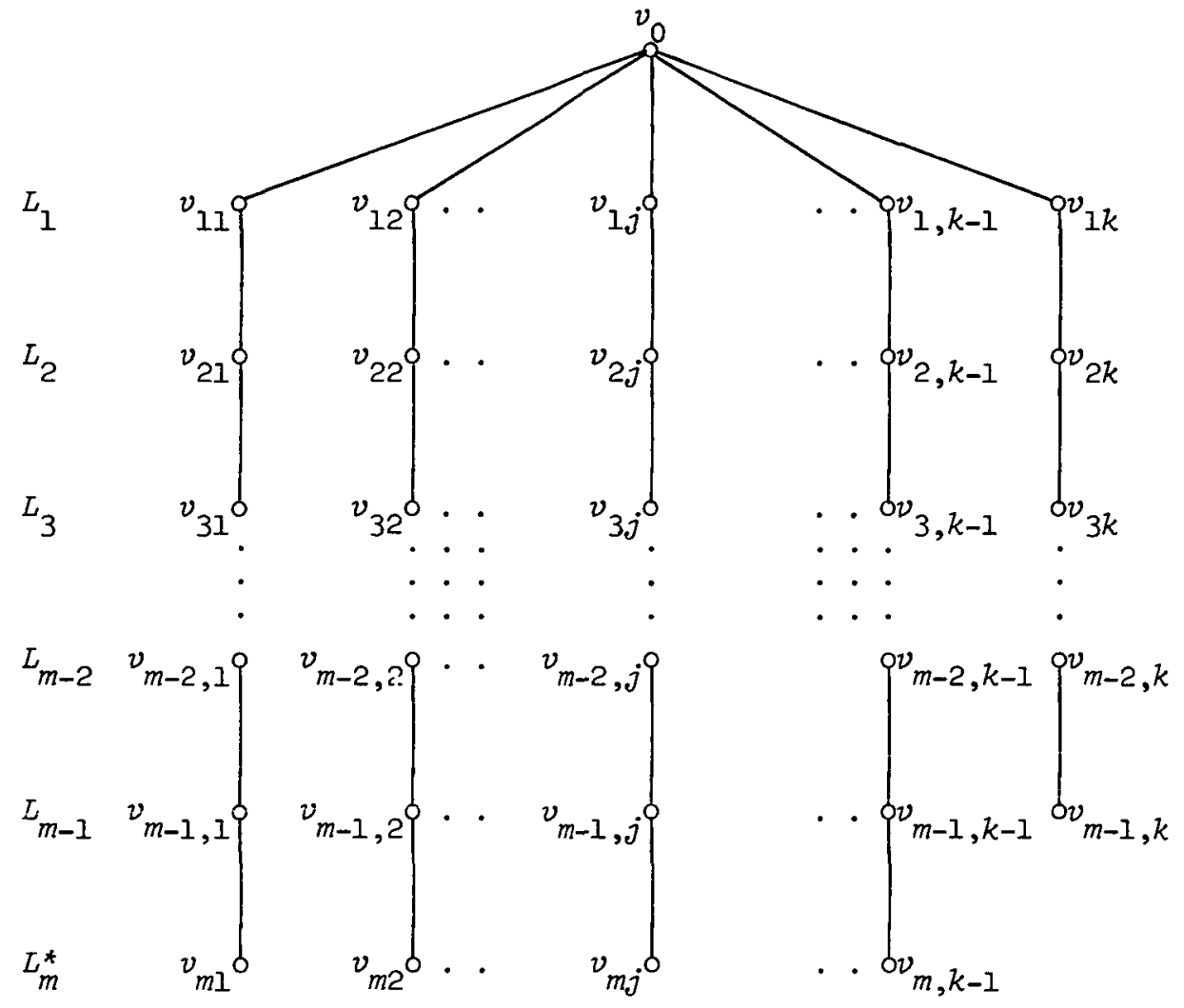

FIGURE 1

With the above terminology, it was proved in Burns et al. [1], that every vertex of $L_{m}$ is adjacent to every vertex of $L_{m-1}$ and to every vertex of $L_{1}$. Repeating the arguments used in Burns et al. [1], and the definition of randomly $k$-axial graphs, with the initial vertex $v$ selected from $L_{1}$, we see that every vertex of $L_{1}$ is adjacent to every vertex of $L_{m}$ and to every vertex of $L_{2}$. Continuing this procedure we conclude that every vertex of $L_{i}$ is adjacent to every vertex of $L_{i-1}$ and to every vertex of $L_{i+1}(i=1,2, \ldots, m)$, where the subscripts are expressed modulo $m$.

For $m \geq 3$ and odd we now show that $G \cong K_{p}$. Our first claim is that every vertex of $L_{i}$ is adjacent to every vertex of $L_{j}, i \neq j$ and 


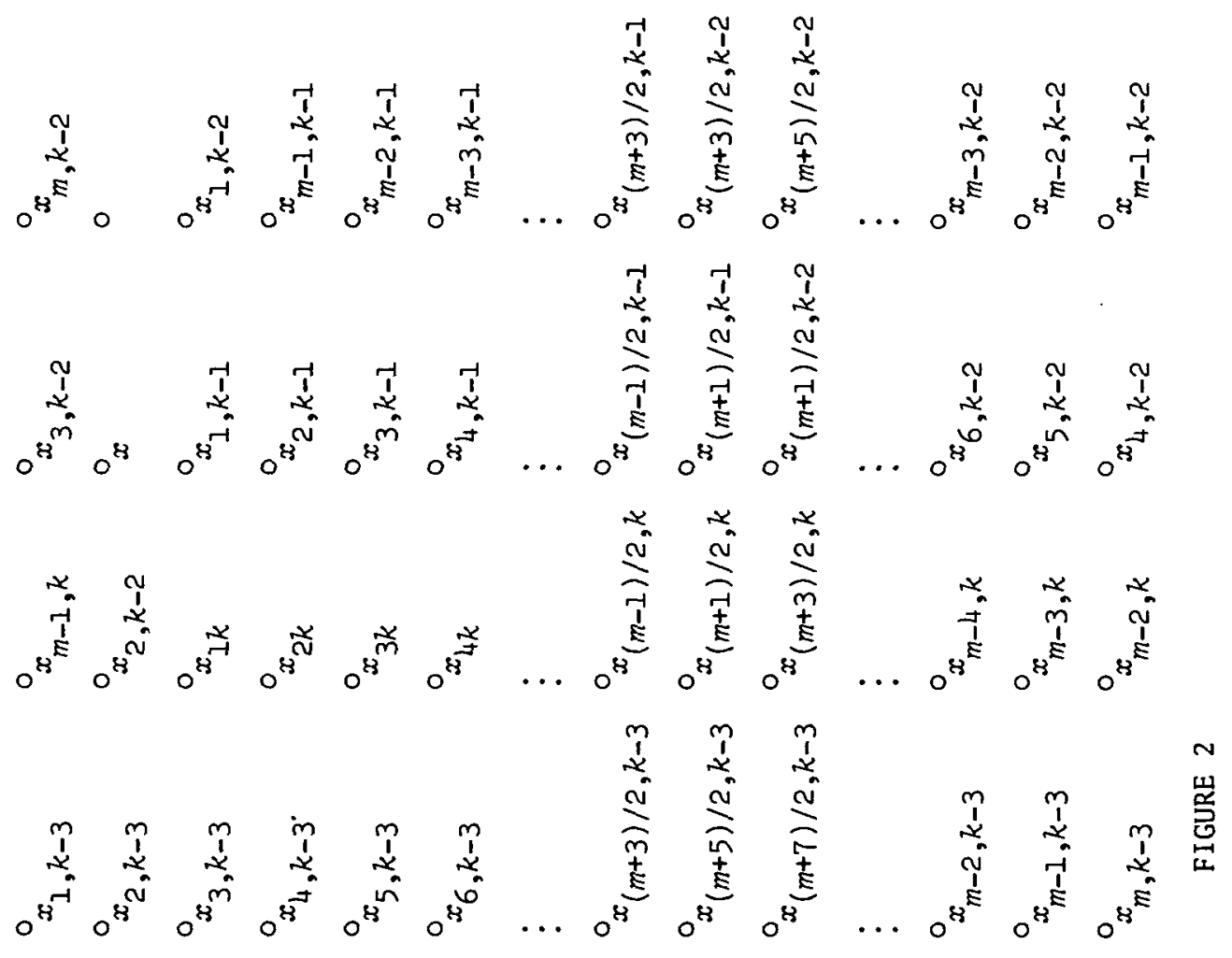

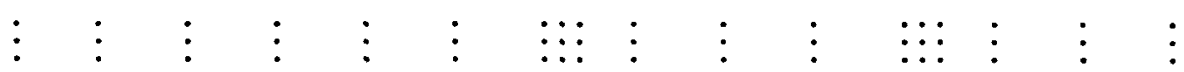

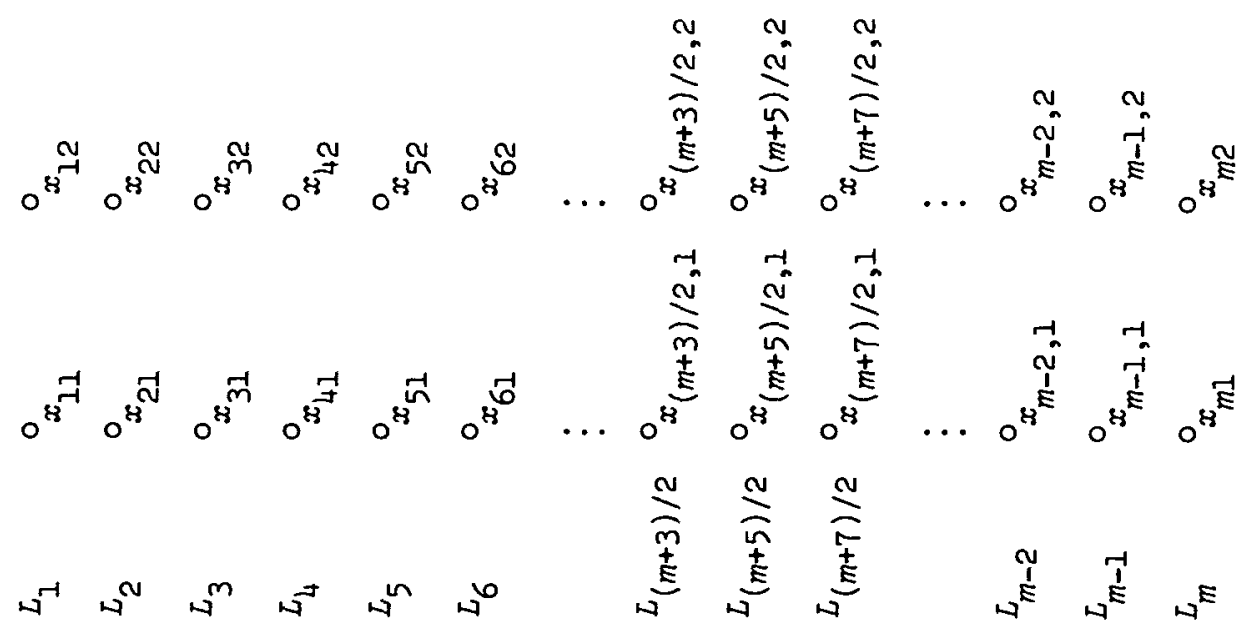


$1 \leq i, j \leq m$. This is already the case for $m=3$. So let $m \geq 5$. We now prove that every vertex of $L_{i}, 1 \leq i \leq m$, is adjacent to every vertex of $L_{i+2}$, where the subscripts are expressed modulo $m$. For convenience, let $x$ denote any vertex of $L_{2}$ (see Figure 2). Using the definition of randomly k-axial graphs with $x$ as the initial vertex, we can obtain a new labelling of the vertices of $G$ as shown in Figure 2 . Note that a vertex of $G$ (in $\left.L_{2}\right)$ has not yet been labelled. Since $G$ is randomly $k$-axial, this vertex must be labelled $x_{m, k-1}$. Since $x_{m, k-1}$ must be adjacent to $x_{m-1, k-1}$ and $x_{m-1, k-1} \in L_{4}$, it follows, because of symmetry, that for each $i \quad(1 \leq i \leq m)$, every vertex of $L_{i}$ is adjacent to every vertex of $L_{i+2}$, where, as always, the subscripts are expressed modulo $m$.

If $m=5$, then our claim holds. If $m \geq 7$, we use the known edges of $G$ and the fact that $G$ is randomly $k$-axial to produce yet another labelling of the vertices of $G$. Relabel vertex $x$ as $y$, vertex $x_{m-1, k-1}$ as $y_{m-2, k-1}$ and vertex $x_{m-2, k-1}$ as $y_{m-1, k-1}$. Every other vertex $x_{r s}$ is relabelled $y_{r s}$. Since $G$ is randomly $k$-axial, the unlabelled vertex in $L_{2}$ must de $y_{m, k-1}$, and is adjacent to $y_{m-1, k-1}$. By symmetry, we conclude that every vertex of $L_{i}$ is adjacent to every vertex of $L_{i+3}$.

If $m=7$, then our claim holds. If $m \geq 9$, we proceed as above to produce yet another labelling of the vertices of $G$. Relabel $y$ as $z$, vertex $y_{m-1, k-1}$ as $z_{m-3, k-1}$ and $y_{m-3, k-1}$ as $z_{m-1, k-1}$. By the same reasoning as above, one can show that every vertex of $L_{i}$ is adjacent to every vertex of $L_{i+4}$ for all $i$. Continuing this procedure, we see that every vertex of $L_{i}$ is adjacent to every vertex of $L_{j}, i \neq j$ and $1 \leq i, j \leq m$.

Finally, we assert that $\left\langle L_{i}\right\rangle$, the subgraph induced by the vertices in $L_{i}$, is complete for $i=1,2, \ldots, m$. Recall that $L_{m}=L_{m}^{*} u\left\{v_{0}\right\}$. 
Consider a relabelling of the vertices of $G$ with $v_{0}$ relabelled as $u$, vertex $v_{m, k-1}$ relabelled as $u_{m-1, k}$ and $v_{m-1, k}$ relabelled as $u_{m, k-1}$. Every other vertex $v_{r s}$ is relabelled $u_{r s}$. Then the vertices $u_{m-1, k-1}$ and $u_{m, k-1}$ are adjacent, that is, $v_{m-1, k-1} v_{m-1, k} \in E(G)$. By symmetry, $\left\langle L_{m-1}\right\rangle$ is complete, and consequently every $\left\langle L_{i}\right\rangle$ is complete.

The preceding result leads to the following immediate consequence for randomly 3-axial graphs.

COROLLARY. If $G$ is a randomly 3-axial graph of order $p$, where $p \equiv 0(\bmod 3)$, then either

(i) $G \cong K_{p}$, or

(ii) $G \cong K(p / 2, p / 2)$ when $p \equiv 0(\bmod 6)$.

Next, let $G$ be a graph of order $p$ which is randomly 3-axial with $p \equiv 2(\bmod 3)$. We may write $p=3 m+2$ where $m \geq 1$. Let $v_{0} \in V(G)$. Since $G$ is randomly 3-axial, $\delta(G) \geq 3$, and we can select three edges incident with $v_{0}$ to produce three paths in $G$ of length one having initial vertex $v_{0}$, and cyclically randomly extend these to obtain a spanning subgraph of $G$ (see Figure $3(a)$ ). Relabel the vertices of $G$ as follows (where $a \rightarrow b$ denotes that $a$ is relabelled $b$ ):

$$
v_{i 2} \rightarrow x_{i 1}, v_{i 1} \rightarrow x_{i 2}, v_{i 3} \rightarrow x_{i 3} \text { for } 1 \leq i \leq m \text {, }
$$

and

$$
v_{0} \rightarrow x \text { and } u \rightarrow u
$$

We observe that $u$ and $x_{m l}$ must be adjacent, that is, $u v_{m 2} \in E(G)$. Similarly $u v_{m 3} \in E(G)$. Thus, a spanning subgraph (as indicated in Figure $3(\mathrm{~b})$ ) may be associated with any vertex $v$ of $G$, where $f$ will denote the final vertex on the first path, and $\left\{f_{1}, f_{2}, f_{3}\right\}$ will be a set of vertices on the three paths which are adjacent to $f$.

Clearly $G$ contains cycles. Let $c: u, u_{1}, u_{2}, \ldots, u_{k-1}, u_{k}=u$ be a cycle in $G$ of minimum length $k$. Since $G$ is randomly 3-axial, 
194 Yousef Alavi, Sabra S. Anderson, Gary Chartrand and S.F. Kapoor

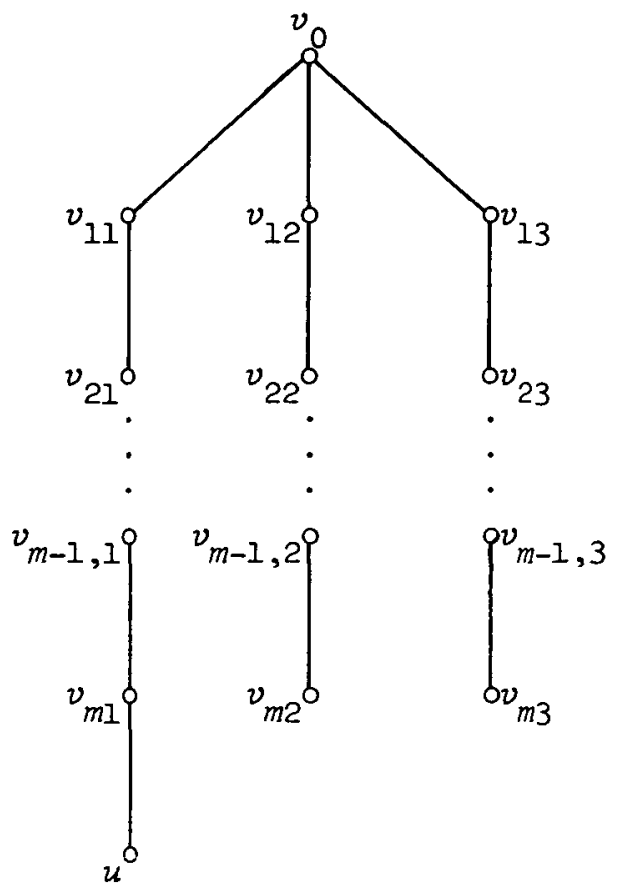

(a)

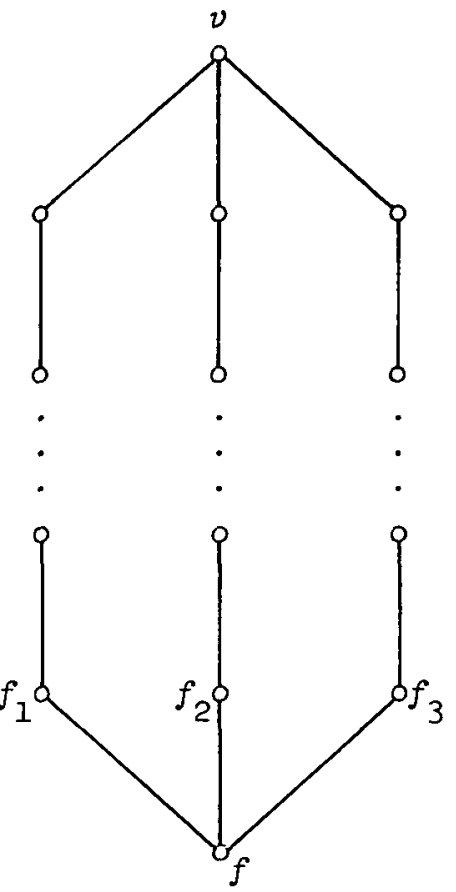

(b)

FIGURE 3

$\delta(G) \geq 3$, and there exists a vertex $w_{1}$ different from $u_{1}$ and $u_{k-1}$ such that uw $_{1} \in E(G)$. Let $P=\left(P_{1}, P_{2}, P_{3}\right)$ be an ordered collection of three internally disjoint paths with $P_{1}: u u_{1}, P_{2}: u u_{k-1}$ and $P_{3}: v a w_{1}$. Consider a cyclic random extension of $P$ where the random extensions of the paths $P_{1}, P_{2}$ and $P_{3}$ result in the following sequence of ordered collections of internally disjoint paths (where $\alpha=[k / 2]$ ):

$$
\begin{gathered}
\left(u u_{1} u_{2}, u u_{k-1}, v a w_{1}\right) \\
\left(u u_{1} u_{2}, u u_{k-1} u_{k-2}, v a w_{1}\right) \\
\left(u u_{1} u_{2}, u u_{k-1} u_{k-2}, v w_{1} w_{2}\right)
\end{gathered}
$$

where the last term of this sequence is

$$
\left(u u_{1} \cdots u_{\alpha}, u u_{k-1} \cdots u_{k-\alpha}, v w_{1} \ldots w_{\alpha}\right) \text { for } k \text { odd }
$$


and where the last term is

$$
\left(u u_{1} \ldots u_{\alpha}, u u_{k-1} \cdots u_{k-\alpha+1}, u w_{1} \ldots w_{\alpha-1}\right) \text { for } k \text { even. }
$$

Observe that $V(C) \cap\left\{w_{1}, w_{2}, \ldots, w_{\alpha}\right\}=\emptyset$ because the cycle $C$ has minimum length $k$. Now $P$ can be cyclically randomly extended in this manner to produce three internally disjoint paths which contain all the vertices of $G$. In view of the preceding remarks we can consider the cycles $C$ and $C^{\prime}$ as shown in Figure 4 . Comparing the lengths of the cycles $C$ and $C^{\prime}$ we obtain

$$
\alpha \leq \begin{cases}(m+1) / 2 & \text { for } k \text { odd }, \\ (m+2) / 2 & \text { otherwise. }\end{cases}
$$
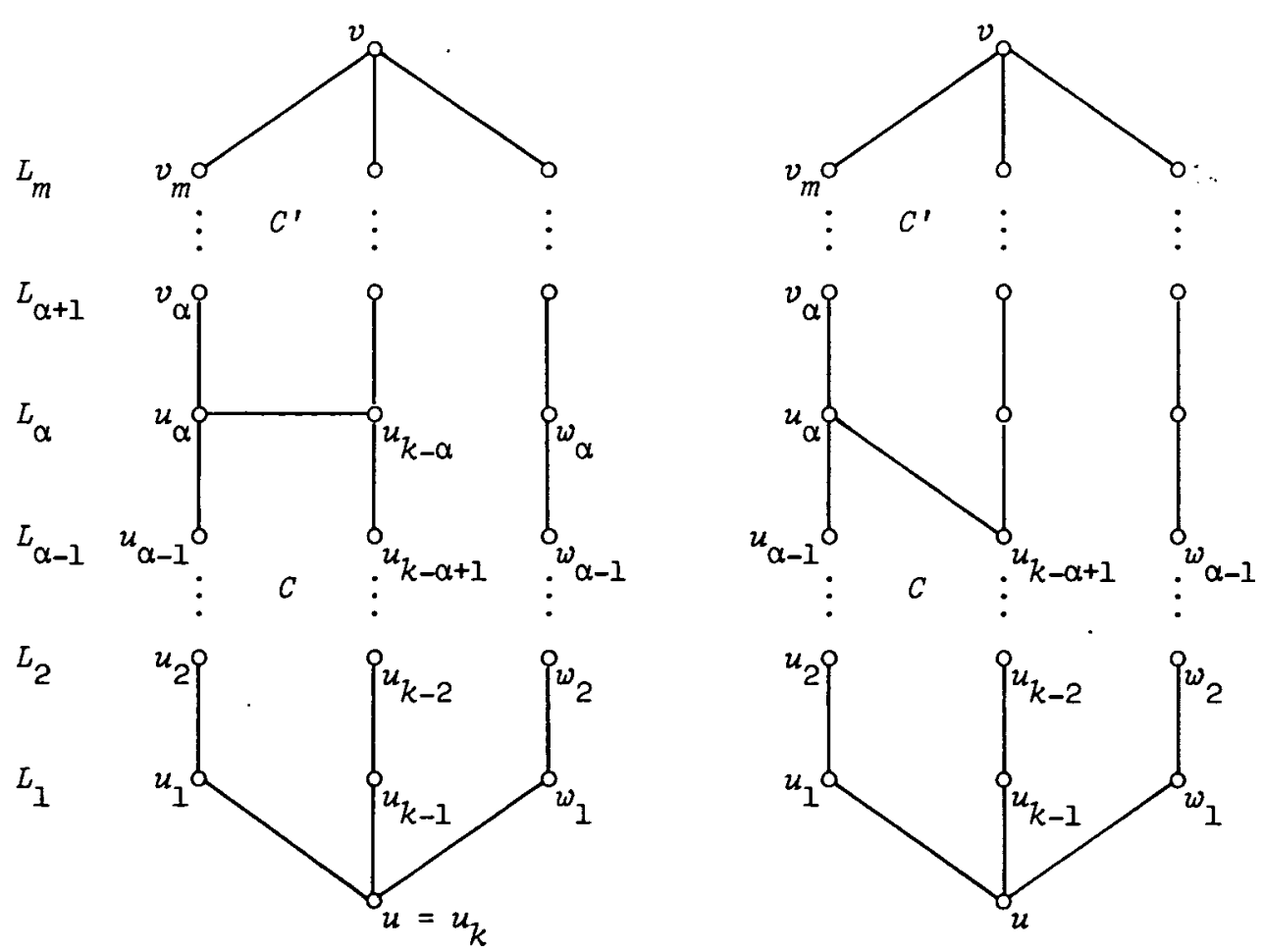

$k$ odd

$\alpha=[k / 2]$

$k$ even

(a)

(b)

FIGURE 4 
It is our objective to show that $k=3$ or $k=4$. Towards this end we assume $k \geq 5$, and obtain a contradiction to the minimality of $k$ by producing a cycle of smaller length. We consider two cases according to the parity of $k$.

$$
\text { Case 1. Let } k=2 \alpha+1 \geq 5 \text {. }
$$

Applying the definition of randomly 3-axial graphs with the vertex $u_{k-\alpha}$ (see Figure $4(\mathrm{a})$ ) as the initial vertex, we can relabel the vertices and consider the following internally disjoint paths (see Figure 5):

$$
\left\{\begin{array}{l}
P_{1}: s_{11} s_{21} \cdots s_{m-\alpha+1,1} s_{m-\alpha+2,1} \\
P_{2}: s s_{12^{s} 22} \cdots s_{m-\alpha+1,2} s_{m-\alpha+2,2} \\
P_{3}: s s_{13^{s}} \cdots s_{m-\alpha+1,3}
\end{array}\right.
$$

Moreover the sets $A=\left\{a_{1}, a_{2}, \ldots, a_{2 \alpha-3}\right\}$ and $B=\left\{b_{1}, b_{2}, \ldots, b_{\alpha-1}\right\}$ are also indicated in Figure 5, with $|A|=2 \alpha-3$ and $|B|=\alpha-1$. If $s_{m-\alpha+2,3} \in A$, then the cycle

$$
s_{m-\alpha+1,3^{s} m-\alpha+2,3} \cdots s_{m-\alpha+2,1} s_{m-\alpha+1,1} s_{m-\alpha+1,3}
$$

has length less than $k_{1}$. So $s_{m-\alpha+2,3} \in B$. Suppose $s_{m-\alpha+2,3}=b_{t}$, where $1 \leq t \leq \alpha-1$.

Let $t=1$. Consider the following internally disjoint paths:

$$
\begin{aligned}
& P_{1}: s s_{11} s_{21} \cdots s_{m-\alpha+2,1} a_{1} a_{2} \cdots a_{\alpha-2}, \\
& P_{2}: s s_{12} s_{22} \cdots s_{m-\alpha+2,2^{a} 2 \alpha-3^{a}} a_{2 \alpha-4} \cdots a_{\alpha}, \\
& P_{3}: s s_{13} s_{23} \cdots s_{m-\alpha+1,3} b_{1} b_{2} \cdots b_{\alpha-1} .
\end{aligned}
$$

In view of Figure $3(b)$, the vertex $a_{\alpha-1}(=f)$ must be adjacent to $b_{\alpha-1}$. We may observe that with

$$
\begin{aligned}
& Q_{1}: a_{\alpha-1} a_{\alpha-2} \cdots a_{2} a_{1} s_{m-\alpha+2,1} \cdots s_{21} s_{11}, \\
& Q_{2}: a_{\alpha-1} a_{\alpha} \cdots a_{2 \alpha-4} a_{2 \alpha-3} s_{m-\alpha+2,2} \cdots s_{22} s_{12},
\end{aligned}
$$

and 

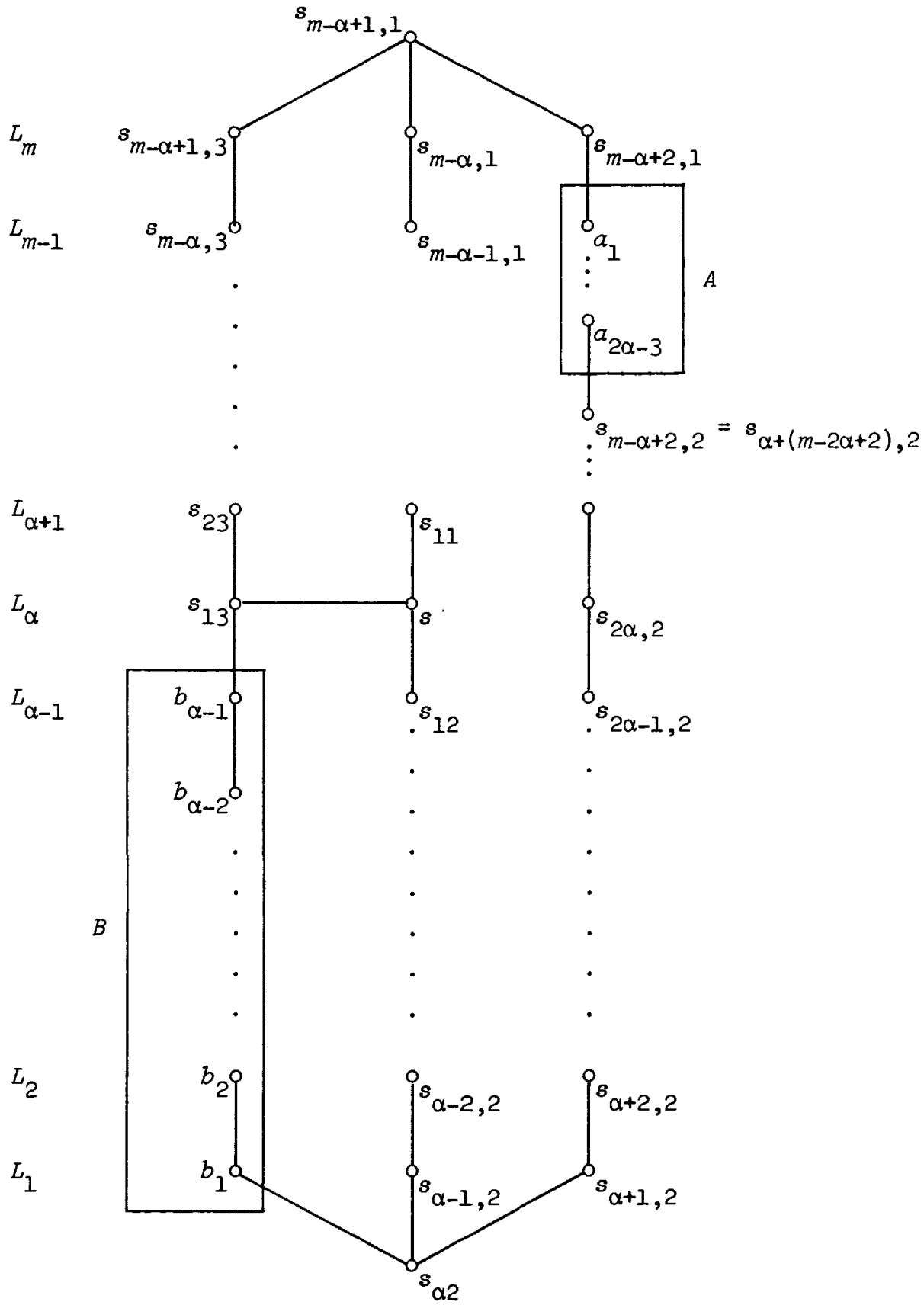

FIGURE 5 


$$
Q_{3}: a_{\alpha-1} b_{\alpha-1} s_{13} s_{23} \cdots s_{m-\alpha+1,3} b_{1} b_{2} \cdots b_{\alpha-2} \text {, }
$$

a similar argument yields $s b_{\alpha-2} \in E(G)$. But then $s_{13^{b-1}} b_{\alpha-2} s$ would be a 4 -cycle in $G$.

In an analogous manner, $t=\alpha-1$ also produces a 4-cycle. Therefore we assume $2 \leq t \leq \alpha-2$ and $k \geq 9$. Now we can obtain random extensions of the paths $P_{1}, P_{2}$ and $P_{3}$ of (1) as follows (see Figure 5):

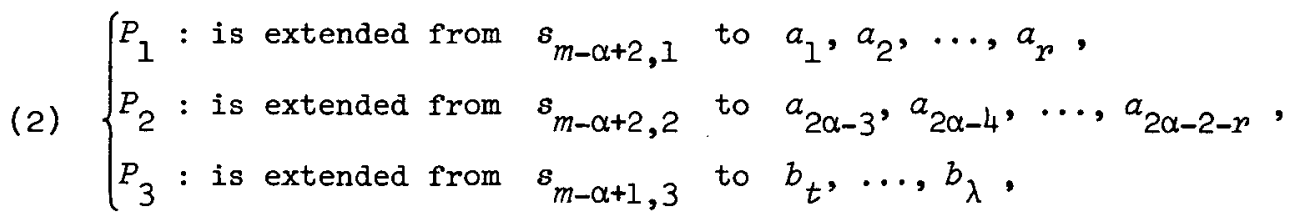
where

$$
\lambda= \begin{cases}1 \quad \text { if } \alpha<2 t, \\ \alpha-1 \text { otherwise, }\end{cases}
$$

and

$$
r= \begin{cases}t \quad \text { if } \alpha<2 t, \\ \alpha-t & \text { otherwise. }\end{cases}
$$

Once the path $P_{3}$ has been extended to $b_{\lambda}$, because of the minimality of $k$, this path cannot be further extended to any remaining vertices in $B$. So there exists at least one vertex

$$
a_{r+c} \in\left\{a_{r+1}, a_{r+2}, \ldots, a_{2 \alpha-3-r}\right\}=\hat{A}, 1 \leq c \leq 2 \alpha-3-2 r,
$$

to which the path $P_{3}$ is extended. Let $\hat{B}$ denote the set of vertices in $B$ that do not already belong to the path $P_{3}$ in (2).

We now observe that the paths $P_{1}, P_{2}$ and $P_{3}$ have their current terminal vertices $a_{r}, a_{2 \alpha-2-r}$ and $a_{r+c}$ in the set $\hat{A} \cup\left\{a_{r}, a_{2 \alpha-2-r}\right\} \subseteq A$. Moreover it is clear that any further cyclic random extensions of $P_{1}$ and $P_{2}$ in $\hat{A}$ must successively proceed to $a_{r+1}, a_{p+2}, \ldots$ for $P_{1}$ and $a_{2 \alpha-3-r}, a_{2 \alpha-4-r}, \ldots$ for $P_{2}$; otherwise, 
if any vertices are bypassed, then a cycle of length less than $k$ results. Let $\beta=\{(2 \alpha-3-2 r) / 2\}=\alpha-2-r$. Suppose $1 \leq c \leq \beta$. Consider the following cyclic random extensions from (2):

$$
\begin{aligned}
& P_{1} \text { : is extended from } a_{r} \text { to } a_{p+1} a_{p+2} \ldots, \\
& P_{2} \text { : is extended from } a_{2 \alpha-2-r} \text { to } a_{2 \alpha-3-r} \cdots,
\end{aligned}
$$

and

$$
P_{3}: \text { is extended from } b_{\lambda} \text { to } a_{r+c} a_{r+c-1} \cdots \text {. }
$$

Then the paths $P_{1}$ and $P_{3}$ eventually extend to adjacent vertices, and subsequent extensions require $P_{1}\left(P_{3}\right)$ to extend to $\hat{B}$ if $c$ is odd (even). Consequently the graph $\left\langle B \cup\left\{a_{p}, a_{p+1}, \ldots, a_{p+c}\right\}\right\rangle$ contains $a$ cycle of length at most

$$
\begin{aligned}
\left|B \cup\left\{a_{r}, a_{r+1}, \ldots, a_{p+c}\right\}\right| & =(\alpha-1)+(1+c) \\
& =\alpha+c \\
& \leq \alpha+(\alpha-2-r) \\
& <2 \alpha+1 .
\end{aligned}
$$

For $\beta<c \leq 2 \alpha-3-2 r, P_{3}$ is extended from $b_{\lambda}$ to $a_{r+c} a_{r+c+1} \cdots$. By arguments similar to the above, subsequent extensions require $P_{2}\left(P_{3}\right)$ to extend to $\hat{B}$ if $c$ is even (odd), leading to a cycle of length less than $k$ in the graph $\left(B \cup\left\{a_{r+c}, a_{r+c+1}, \ldots, a_{2 \alpha-3-r}, a_{2 \alpha-2-r}\right\}\right)$.

Case 2. Let $k=2 \alpha \geq 6$.

We use the spanning subgraph of $G$ as shown in Figure 4 (b) and apply the techniques of the preceding case to obtain a cycle in $G$ of length less than $k$. The details are omitted.

The following result can now be stated.

LEMMA 1. Let $G$ be a randomly 3-axial graph of order $p$, where $p \equiv 2(\bmod 3)$. Then the length of a smallest cycle in $G$ is 3 or 4 .

In the next two results we consider randomly 3-axial graphs of order $p \equiv 2(\bmod 3)$ where the length of a smallest cycle is 3 or 4 .

LEMMA 2. Let $G$ be a randomly 3-axial graph of order $p$, where 
$p \equiv 2(\bmod 3)$. If $G$ contains $K_{3}$ as a subgraph, then $G \cong K_{p}$.

Proof. In view of Figure 4 (a) the graph $G$ contains a subgraph as shown in Figure 6. The levels $L_{t}=\left\{u_{t 1}, u_{t 2}, u_{t 3}\right\}, 1 \leq t \leq m$, are also indicated in Figure 6 . Consider the following path with initial vertex $u_{11}$ :

$$
\begin{gathered}
u_{11} u_{21} \cdots u_{m 1} v, \\
u_{11} u_{12} u_{22} \cdots u_{m 2},
\end{gathered}
$$

and

$$
u_{11} u u_{13} u_{23} \cdots u_{m-1,3}
$$

(Recall the notation introduced in Figure 3 (b).) Then $f f_{2}=u_{m 3} u_{m 2} \in E(G)$. By analogous arguments, and in view of the symmetry, we see that $\left\langle u_{11}, u_{12}, u_{13}\right\rangle \cong\left\langle u_{m 1}, u_{m 2}, u_{m 3}\right\rangle \cong K_{3}$. Now consider the paths

$$
\begin{aligned}
& u_{11} u_{21} \cdots u_{m 1} v, \\
& u_{11} u_{12} \cdots u_{m 2},
\end{aligned}
$$

and

$$
u_{11} u_{13} \cdots u_{m 3}
$$

with $f f_{1}=u v \in E(G)$ and $f f_{2}=u u_{m 2} \in E(G)$. By symmetry, we also conclude that $u$ is adjacent with every vertex in level $L_{m}$ and $v$ is adjacent with every vertex in level $L_{1}$. It can be easily seen that if $m=1$, then $G \cong K_{5}$. So let $m \geq 2$. Observe that $\left\langle u, v, L_{m}\right\rangle \cong K_{5}$. The paths

$$
\begin{aligned}
& u u_{11} u_{21} \cdots u_{m 1}, \\
& u v u_{m 2} \cdots u_{22},
\end{aligned}
$$

and

$$
u_{13} u_{23} \cdots u_{m 3}
$$


yield $f f_{1}=u_{12} u_{m l} \in E(G)$. Again, by symmetry, every vertex in $L_{1}$ is adjacent with every vertex in $L_{m}$. Thus $\left\langle u, v, L_{m}, L_{1}\right\rangle \cong K_{8}$. We now assume $m \geq 3$.

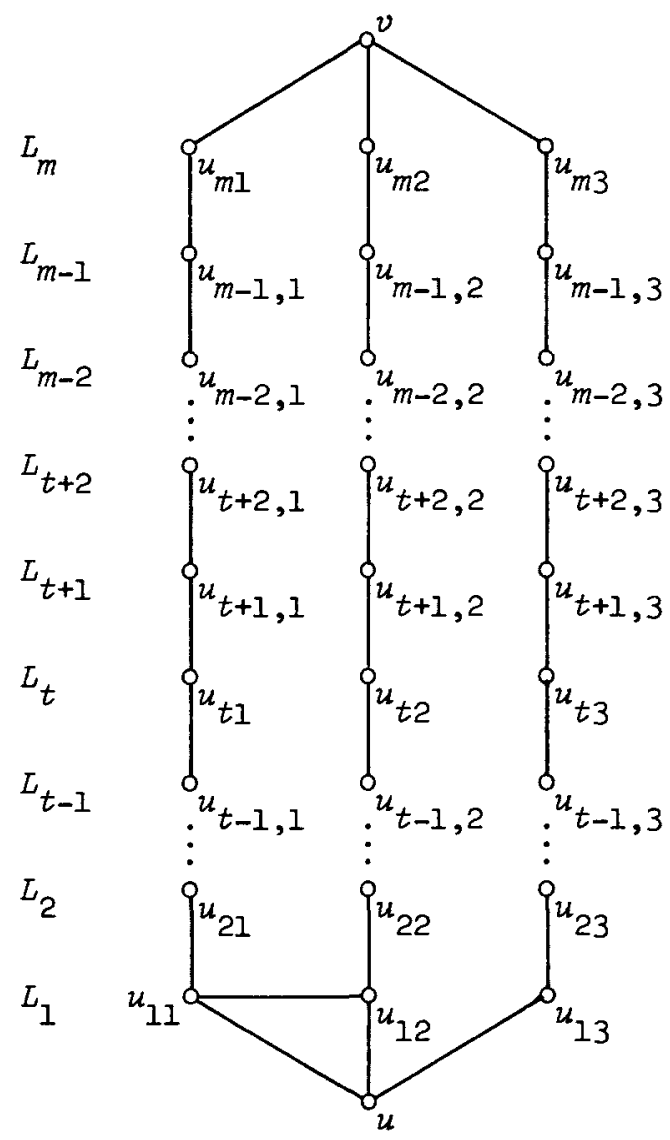

FIGURE 6

Next, we claim that $\left\langle u, v, L_{m}, L_{1}, L_{2}, \ldots, L_{t}\right) \cong K_{3(t+1)+2}$, for all $t$ satisfying $1 \leq t \leq m-1$. The result already holds when $t=1$. Assume that $\left\langle u, v, L_{m}, L_{1}, L_{2}, \ldots, L_{t}\right\rangle \cong K_{3(t+1)+2}$ for any positive integer $t$, where $1 \leq t \leq m-2$. Consider the paths

$$
\begin{aligned}
& P_{1}: v u_{t-1,1} u_{t-2,1} \cdots u_{11} u_{m 1} u_{m-1,1} \cdots u_{t 1}, \\
& P_{2}: v u u_{12} u_{22} \ldots u_{t 2} u_{m 2} u_{m-1,2} \cdots u_{t+2,2},
\end{aligned}
$$

and 
202 Yousef Alavi, Sabra S. Anderson, Gary Chartrand and S.F. Kapoor

$$
P_{3}: v u_{13} u_{23} \cdots u_{t 3} u_{m 3} u_{m-1,3} \cdots u_{t+1,3}
$$

where, for $t=1$, we let

$$
P_{1}: v u_{11} u_{m 1} u_{m-1,1} \cdots u_{21} \text {. }
$$

Then the vertex $f=u_{t+1,2}$ is adjacent to $u_{t 1}$ and $u_{t+1,3} \cdot$ By symmetry we conclude that $\left(L_{t+1}\right) \cong K_{3}$ and $\left(L_{t}, L_{t+1}\right) \cong K_{6}$. Similarly, the paths

$$
\begin{aligned}
& v u_{m 1} u_{m-1,1} \cdots u_{11}, \\
& v u_{12} u_{22} \cdots u_{t 2} u_{m 2} u_{m-1,2} \cdots u_{t+2,2},
\end{aligned}
$$

and

$$
v u_{m 3} u_{m-1,3} \cdots u_{13}
$$

produce $f=u_{t+1,2}$, and then, every vertex in $L_{t+1}$ is adjacent with every vertex in $L_{1}$. For $t \geq 2,\left\langle u, v, L_{1}, L_{2}, \ldots, L_{t-1}\right\rangle \cong K_{3(t-1)+2}$ implies that we may consider the vertices in this complete graph interchangeable. So every vertex in level $L_{t+1}$ is adjacent with every vertex in the set $\{u, v\} \cup \underset{i=1}{t-1} L_{i}$. If $t=1$, the paths

$$
\begin{aligned}
& v u_{11} u_{m 1} u_{m-1,1} \cdots u_{21}, \\
& v u_{12} u_{m 2} u_{m-1,2} \cdots u_{22},
\end{aligned}
$$

and

$$
v u_{13} u_{m 3} u_{m-1,3} \cdots u_{23}
$$

show that $f=u$ is adjacent with every vertex in the set $L_{2}$. Similarly, $v$ is also adjacent with every vertex in level $L_{2}$. So every vertex in level $L_{2}$ is adjacent with every vertex in the set $\{u, v\} \cup L_{1}$. Finally, the paths

$$
u u_{11} u_{21} \cdots u_{t 1} v u_{m 1} u_{m-1,1} \cdots u_{t+2,1},
$$




$$
u u_{12} u_{22} \cdots u_{m 2}
$$

and

$$
u u_{13} u_{23} \ldots u_{m 3}
$$

have $f=u_{t+1,1}$. So every vertex in level $L_{t+1}$ is adjacent with every vertex in level $L_{m}$. Consequently

$$
\left\langle u, v, L_{m}, L_{1}, L_{2}, \ldots, L_{t}, L_{t+1}\right\rangle \cong K_{3(t+2)+2},
$$

for $1 \leq t \leq m-2$. Our claim follows by induction, and then, $G \cong\left\langle u, v, L_{m}, L_{1}, \ldots, L_{m-1}\right\rangle \cong K_{p}$.

LEMMA 3. Let $G$ be a randomly 3-axial graph of order $p$, where $p \equiv 2(\bmod 3)$. Let the length of a smallest cycle in $G$ be 4 . Then $p$ is even and $G \cong K(p / 2, p / 2)$.

Proof. We can use Figure 4 (b) to obtain a subgraph of $G$ as shown in Figure 7 (a), together with the levels $L_{i}=\left\{u_{i 1}, u_{i 2}, u_{i 3}\right\}$,

$1 \leq i \leq m$. Using the definition of randomly 3-axial graphs and symmetry, with appropriate choices for initial vertices and collections of internally disjoint paths, we obtain additional edges shown in Figure 7 (b). Similar techniques lead to the following additional results;

$$
\left\langle L_{1} ; L_{2}\right\rangle \cong\left\langle L_{1} ; L_{m}\right\rangle \cong\left\langle L_{m-1} ; L_{m}\right\rangle \cong K(3,3) \text { and } u v \in E(G) \text {. }
$$

Since $p=3 m+2$, if $m=1$, we see that $G$ contains $K_{3}$. If $m=2$, then $G \cong K(4,4)$; and for $m=3,\left(u_{11}, u_{21}, u_{31}\right) \cong K_{3}$. So let $m \geq 4$.

Consider the following relabelings of $V(G)$ :

(i) $u_{i 1} \rightarrow u_{i 1}^{\prime}$ and $u_{i 3}+u_{i 3}^{\prime}$ for $1 \leq i \leq m, u \rightarrow u_{m 2}^{\prime}$, $v \rightarrow u_{m-1,2}^{\prime}, u_{12}+v^{\prime}, u_{22} \rightarrow u^{\prime}$, and $u_{j 2} \rightarrow u_{j-2,2}^{\prime}$ for $3 \leq j \leq m$;

(ii) $u_{i 1} \rightarrow u_{i 1}^{\prime \prime}$ and $u_{i 3} \rightarrow u_{i 3}^{\prime \prime}$ for $1 \leq i \leq m, u \rightarrow u_{22}^{\prime \prime}$, $v \rightarrow u_{12}^{\prime \prime}, u_{m-1,2}+v^{\prime \prime}, u_{m 2} \rightarrow u^{\prime \prime}$, and $u_{j 2}+u_{j+2,2}^{\prime \prime}$ for $I \leq j \leq m-2$. 
204 Yousef Alayi, Sabra S. Anderson, Gary Chartrand and S.F. Kapoor

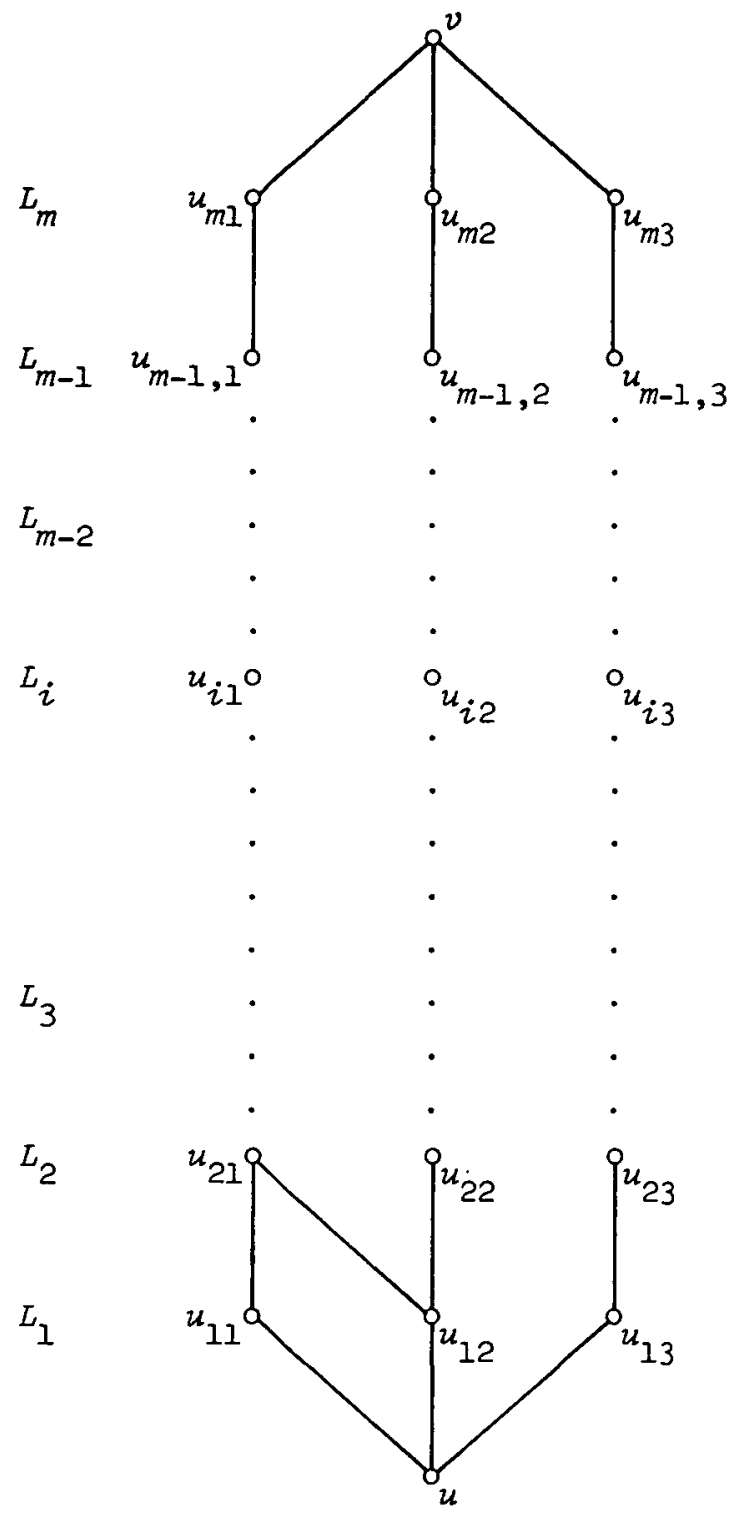

(a)

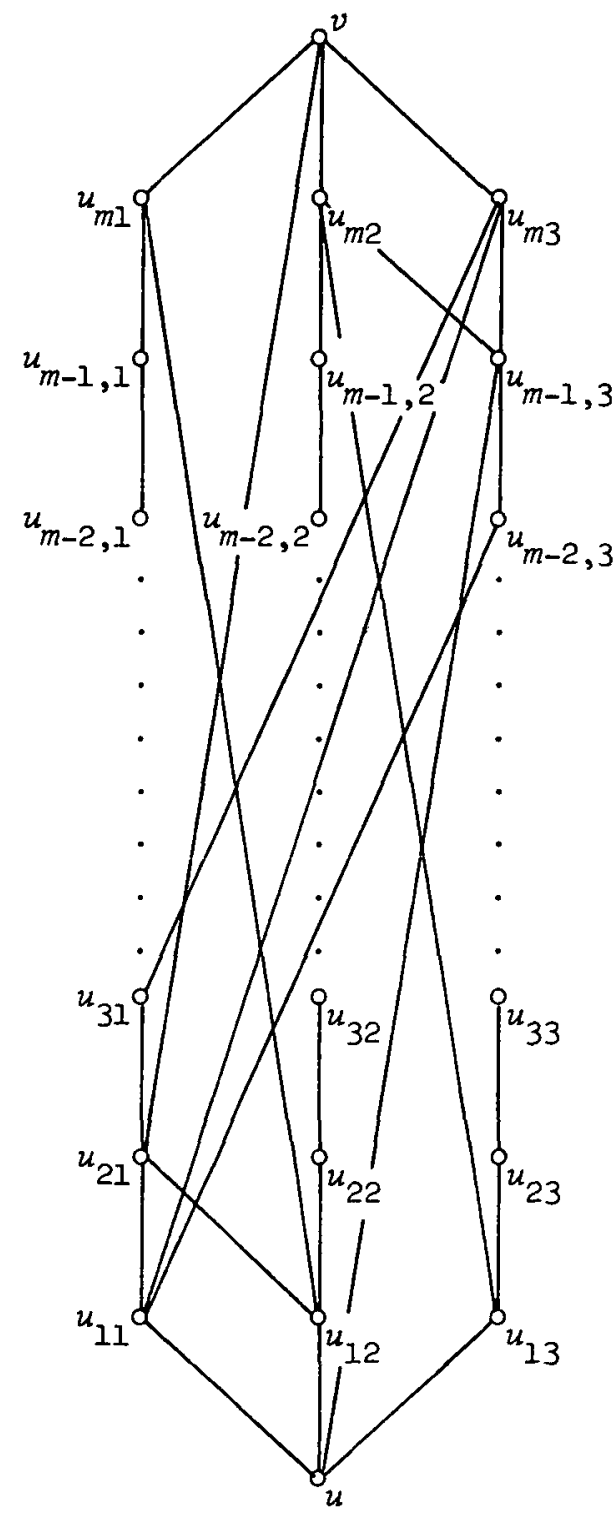

(b)

FIGURE 7

Observe that the edges $u_{11}^{\prime} u_{23}^{\prime}$ and $u_{11}^{\prime \prime} u_{23}^{\prime \prime}$ lead to 4-cycles in $G$ analogous to the 4-cycle shown in Figure 7 (a). Interpreting the results in (3) for the new labellings ( $i$ ) and ( $i i)$ in terms of the original labels, we obtain the following results: 
(4) $\left\langle L_{1} ; L_{4}\right\rangle,\left\langle L_{2} ; L_{3}\right\rangle,\left\langle L_{3} ; L_{4}\right\rangle,\left\langle L_{m-3} ; L_{m-2}\right\rangle,\left\langle L_{m-3} ; L_{m}\right\rangle,\left\langle L_{m-1}, L_{m-2}\right\rangle$ are isomorphic with $K(3,3) ;\left\langle u ; L_{m-1}\right\rangle \cong\left\langle v ; L_{2}\right\rangle \cong K(1,3)$.

If $m=4$, then $G$ contains a subgraph isomorphic with $K(7,7)$. But if any pair of vertices not adjacent in $K(7,7)$ are adjacent in $G$, then $G$ would contain a triangle. So $G \cong K(7,7)$. For $m=5$, $\left\langle L_{2} ; L_{5}\right\rangle \cong K(3,3)$ and $\left\langle v ; L_{2}\right\rangle \cong\left(v ; L_{5}\right\rangle \cong K(1,3)$ leads to a triangle in the graph. For $m \geq 6$ we repeat the above procedure iteratively by interpreting the results in (3) and (4) for the new labellings in terms of the original labels. It may be observed that the nature of the resulting adjacencies between levels forces $m$ to be even (that is, $p$ is even), otherwise, $G$ would contain triangles. Eventually

$$
G \supseteq\left\langle u, L_{2}, L_{4}, \ldots, L_{m} ; v, L_{1}, L_{3}, \ldots, L_{m-1}\right\rangle \cong K(p / 2, p / 2) \text {. }
$$

As before, $G$ cannot contain any edges not already present in the complete bipartite graph. Consequently $G \cong K(p / 2, p / 2)$.

Combining the preceding lemmas we now have the following.

THEOREM 2. If $G$ is a randomly 3-axial graph of order $p$, where $p \equiv 2(\bmod 3)$, then either

(i) $G \cong K_{p}$, or

(ii) $G \cong K(p / 2, p / 2)$ when $p \equiv 2(\bmod 6)$.

It was proved in Burns, et al. [1], that the graph $K(m, n)$ is randomly $k$-axial $(k \geq 3)$ if and only if $m=n$, and $m \equiv 0(\bmod k)$ or $m \equiv 1(\bmod k)$. Thus, for $k=3, K(m, n)$ is randomly 3 -axial if and only if $m=n$, and $m \equiv 0(\bmod 3)$ or $m \equiv 1(\bmod 3)$. Let $G$ be a randomly 3-axial graph of order $p$, where $p \equiv I(\bmod 3)$. If $G \cong K(p / 2, p / 2)$, then we need $p / 2 \equiv 0,1(\bmod 3)$, that is, $p \equiv 0,2(\bmod 3)$, and this contradicts $p \equiv 1(\bmod 3)$. So no complete bipartite graph of order $p \equiv 1(\bmod 3)$ can be randomly 3-axial. For $p=4,7$ and 10 it has been verified that the only graph of order $p$ which is randomly 3 -axial is $K_{p}$, and we conjecture that this is true for all $p \equiv 1(\bmod 3)$. 


\section{References}

[1] David Burns, Gary Chartrand, S.F. Kapoor and Farrokh Saba, "Randomly k-axial graphs", Bulz. Austral. Math. Soc. 23 (1981), 143-156.

[2] Gary Chartrand and Hudson V. Kronk, "Randomly traceable graphs", SIAM J. App 2. Math. 16 (1968), 696-700.

Professors Y. Alavi, G. Chartrand and S.F. Kapoor, Department of Mathematics, Western Michigan University, Kal amazoo, Michigan 49008, USA.

Professor S.S. Anderson, Department of Mathematics, University of Minnesota, Duluth, Minnesota 55812, USA. 